\title{
Characterization of Symmetry Properties of First Integrals for Submaximal Linearizable Third-Order ODEs
}

\author{
K. S. Mahomed and E. Momoniat \\ Differential Equations, Continuum Mechanics and Applications, School of Computational and Applied Mathematics, \\ University of the Witwatersrand, Wits 2050, South Africa \\ Correspondence should be addressed to K. S. Mahomed; komalmajeed@hotmail.com
}

Received 23 August 2013; Accepted 13 September 2013

Academic Editor: Chaudry Masood Khalique

Copyright (C) 2013 K. S. Mahomed and E. Momoniat. This is an open access article distributed under the Creative Commons Attribution License, which permits unrestricted use, distribution, and reproduction in any medium, provided the original work is properly cited.

\begin{abstract}
The relationship between first integrals of submaximal linearizable third-order ordinary differential equations (ODEs) and their symmetries is investigated. We obtain the classifying relations between the symmetries and the first integral for submaximal cases of linear third-order ODEs. It is known that the maximum Lie algebra of the first integral is achieved for the simplest equation and is four-dimensional. We show that for the other two classes they are not unique. We also obtain counting theorems of the symmetry properties of the first integrals for these classes of linear third-order ODEs. For the 5 symmetry class of linear third-order ODEs, the first integrals can have $0,1,2$, and 3 symmetries, and for the 4 symmetry class of linear third-order ODEs, they are 0,1 , and 2 symmetries, respectively. In the case of submaximal linear higher-order ODEs, we show that their full Lie algebras can be generated by the subalgebras of certain basic integrals.
\end{abstract}

\section{Introduction}

Algebraic properties of first integrals of scalar-order differential equations have been of interest in the recent literature since the early works of Lie $[1,2]$ on symmetries and invariants of ODEs. The Noether classification has also drawn attention to them in [3]. The symmetry classification of scalar ordinary differential equations has been studied in recent years (see, e.g., $[4,5])$. Of the algebraic properties, the maximal symmetry properties of first integrals of linear ODEs have attracted particular attention. In [6], the authors showed that the full Lie algebra $\mathrm{sl}(3, R)$ of scalar linear second-order ODEs represented by the simplest free particle equation can be generated by the three triplets of the three-dimensional algebras of the two basic integrals and their quotient. In their work [4], they found that the symmetries of the maximal cases of scalar linear $n$ th-order ODEs, $n \geq 3$, are $n+1, n+2$, and $n+4$. Thus, for scalar linear third-order equations these correspond to 4, 5, and 7 symmetries. Govinder and Leach studied the symmetry properties of first integrals of scalar linear third-order ODEs which belong to these three classes in [7]. They showed that the three equivalence classes each has certain first integrals with a specific number of point symmetries. Later Flessas et al. in [8] examined the symmetry structure of the first integrals of higher-order equations of maximal symmetry and they proved some interesting basic propositions related to the scaling symmetry and basic integrals.

In a recent paper [9], Mahomed and Momoniat, obtained a classifying relation between the symmetries and the first integrals of linear or linearizable scalar second-order ODEs. They presented a complete classification of point symmetries of first integrals of such linear ODEs, and as a consequence, they provided a counting theorem for the point symmetries of first integrals of scalar linearizable second-order ODEs. They showed that there exist the $0,1,2$, or 3 point symmetry cases and that the maximal algebra case is unique. These authors then considered the problem of classifying the symmetry property of the first integrals of the simplest thirdorder equation $y^{\prime \prime \prime}=0$ in the paper [10]. They found that the maximal Lie algebra of a first integral for this equation is unique and four-dimensional. They also showed that the Lie algebra of the simplest linear third-order equation is generated by the symmetries of two basic integrals instead of three. Moreover, they obtained counting theorems of 
the symmetry properties of the first integrals for such linear third-order ODEs of maximal symmetry. Furthermore, they provided insights into the manner in which one can generate the full Lie algebra of higher-order ODEs of maximal symmetry from two of their basic integrals.

The discussion of this work is about the Lie algebraic properties of first integrals of scalar linearizable third-order ODEs of the submaximal classes which are represented by $y^{\prime \prime \prime}-y^{\prime}=0$ and $y^{\prime \prime \prime}+f(x) y^{\prime \prime}-y^{\prime}-f(x) y=0$, where $f(x)$ is an arbitrary function of $x$. The former has four-point symmetries, and the latter has five. As we mentioned earlier, there was some work [8] done by Flessas et al. for the simplest class and extended by Mahomed and Momoniat in [10] to provide a complete analysis on the symmetries and first integrals for this simplest class of ODEs which included the maximal algebra case being generated by algebras of two basic integrals of the equation. In the present study, we deduce the classifying relation between the point symmetries and first integrals for the submaximal classes of scalar linear third-order equations. Then, by using this, we find the point symmetry properties of the first integrals of the submaximal classes of third-order equations $y^{\prime \prime \prime}-y^{\prime}=0$ and $y^{\prime \prime \prime}+$ $f(x) y^{\prime \prime}-y^{\prime}-f(x) y=0$ which also represent all linearizable by point transformations third-order ODEs that reduce to these classes. We obtain counting theorems for the number of point symmetries possessed by an integral of such equations. Noteworthy is that the maximal algebra is not unique.

In the next section, we study the point symmetry properties of the integrals of the 4 symmetry class represented by $y^{\prime \prime \prime}-y^{\prime}=0$. This section is to remind the reader under what conditions point symmetries of first integrals of scalar linear third-order ODEs exist [10]. Then, in Section 3 we analyze the class $y^{\prime \prime \prime}+f(x) y^{\prime \prime}-y^{\prime}-f(x) y=0$ which has four-point symmetries for the symmetry structure of its first integrals. In Section 4, we focus on the generation of the full algebra by subalgebras of certain basic integrals. The Conclusion contains a summary and hints for future work.

\section{Algebraic Properties of the Integrals of $y^{\prime \prime \prime}-y^{\prime}=0$}

We consider the representative third-order ODE

$$
y^{\prime \prime \prime}-y^{\prime}=0
$$

which has five-point symmetries

$$
\begin{aligned}
& X_{1}=\frac{\partial}{\partial x}, \\
& X_{2}=\frac{\partial}{\partial y}, \\
& X_{3}=e^{x} \frac{\partial}{\partial y}, \\
& X_{4}=e^{-x} \frac{\partial}{\partial y}, \\
& X_{5}=y \frac{\partial}{\partial y} .
\end{aligned}
$$

The ordering of these is the translation in $x$ followed by the three solution symmetries and then the homogeneity symmetry. It is easy to see here that (1) has three functionally independent first integrals

$$
\begin{aligned}
& I_{1}=y^{\prime \prime}-y, \\
& I_{2}=e^{x} y^{\prime \prime}-e^{x} y^{\prime}, \\
& I_{3}=e^{-x} y^{\prime \prime}+e^{-x} y^{\prime} .
\end{aligned}
$$

The order of the integrals is dictated by their algebraic properties which come at the end of this section.

2.1. Classifying Relation for the Symmetries of $y^{\prime \prime \prime}-y^{\prime}=0$. Let $F$ be an arbitrary function of $I_{1}, I_{2}$, and $I_{3}$; namely, $F=$ $F\left(I_{1}, I_{2}, I_{3}\right)$. The symmetry of this general function of the first integrals is

$$
X^{[2]} F=X^{[2]} I_{1} \frac{\partial F}{\partial I_{1}}+X^{[2]} I_{2} \frac{\partial F}{\partial I_{2}}+X^{[2]} I_{3} \frac{\partial F}{\partial I_{3}}=0
$$

where

$$
\begin{aligned}
X^{[2]} I_{1} & =\left[\xi \frac{\partial}{\partial x}+\eta \frac{\partial}{\partial y}+\zeta_{x} \frac{\partial}{\partial y^{\prime}}+\zeta_{x x} \frac{\partial}{\partial y^{\prime \prime}}\right]\left(y^{\prime \prime}-y\right) \\
& =-\eta+\zeta_{x x}, \\
X^{[2]} I_{2} & =\left[\xi \frac{\partial}{\partial x}+\eta \frac{\partial}{\partial y}+\zeta_{x} \frac{\partial}{\partial y^{\prime}}+\zeta_{x x} \frac{\partial}{\partial y^{\prime \prime}}\right]\left(e^{x} y^{\prime \prime}-e^{x} y^{\prime}\right) \\
& =\left(e^{x} y^{\prime \prime}-e^{x} y^{\prime}\right) \xi-e^{x} \zeta_{x}+e^{x} \zeta_{x x}, \\
X^{[2]} I_{3} & =\left[\xi \frac{\partial}{\partial x}+\eta \frac{\partial}{\partial y}+\zeta_{x} \frac{\partial}{\partial y^{\prime}}+\zeta_{x x} \frac{\partial}{\partial y^{\prime \prime}}\right]\left(e^{-x} y^{\prime \prime}+e^{-x} y^{\prime}\right) \\
& =\left(-e^{-x} y^{\prime \prime}-e^{-x} y^{\prime}\right) \xi+e^{-x} \zeta_{x}+e^{-x} \zeta_{x x} .
\end{aligned}
$$

Now $\xi, \eta, \zeta_{x}$, and $\zeta_{x x}$ are

$$
\begin{gathered}
\xi=a_{1}, \\
\eta=a_{2}+e^{x} a_{3}+e^{-x} a_{4}+y a_{5}, \\
\zeta_{x}=e^{x} a_{3}-e^{-x} a_{4}+y^{\prime} a_{5}, \\
\zeta_{x x}=e^{x} a_{3}+e^{-x} a_{4}+y^{\prime \prime} a_{5} .
\end{gathered}
$$

These are the coefficients of $X^{[2]}$ which are obtained by

$$
X^{[2]}=\sum_{i=1}^{5} a_{i} X_{i}^{[2]},
$$

where $X_{i}$ s are the symmetry generators as given in (2) and the $a_{i} s$ are constants. The reason for taking a linear combination is that the symmetries of the first integrals are always the symmetries of the equation (see [11] for a general result on this). 
After substitution of the values of $X^{[2]} I_{1}, X^{[2]} I_{2}$, and $X^{[2]} I_{3}$ given in (5), with $\xi, \eta, \zeta_{x}$, and $\zeta_{x x}$ as in (6) and together using the first integrals $I_{1}=y^{\prime \prime}-y, I_{2}=e^{x} y^{\prime \prime}-e^{x} y^{\prime}$, and $I_{3}=e^{-x} y^{\prime \prime}+e^{-x} y^{\prime}$ in (4), we finally arrive at the classifying relation

$$
\begin{aligned}
& \left(-a_{2}+I_{1} a_{5}\right) \frac{\partial F}{\partial I_{1}}+\left[\left(a_{1}+a_{5}\right) I_{2}+2 a_{4}\right] \frac{\partial F}{\partial I_{2}} \\
& +\left[\left(a_{5}-a_{1}\right) I_{3}+2 a_{3}\right] \frac{\partial F}{\partial I_{3}}=0 .
\end{aligned}
$$

The relation (8) provides the relationship between the symmetries and first integrals of the third-order equation (1). We use this relation in order to classify the first integrals according to their symmetries.

2.2. Symmetry Structure of the First Integrals of $y^{\prime \prime \prime}-y^{\prime}=0$. We utilize the classifying relation (8) to investigate the number and properties of the symmetries of the first integrals of the ODE (1).

In the first instance we see that if $F$ is arbitrary, then by use of (8) we immediately see that

$$
\begin{gathered}
-a_{2}+I_{1} a_{5}=0, \\
\left(a_{1}+a_{5}\right) I_{2}+2 a_{4}=0, \\
\left(a_{5}-a_{1}\right) I_{3}+2 a_{3}=0 .
\end{gathered}
$$

The relations in (9) imply that all the $a$ 's are zero. Hence, there is no symmetry for this case, that is, for $F$ an arbitrary function.

In order to effectively and systematically study the one and higher symmetry cases of first integrals, we obtain optimal systems of one-dimensional subalgebra spanned by (2). Then, we invoke the classifying relation (8). So the strategy followed here is different from that employed for the simplest third-order ODE, $y^{\prime \prime \prime}=0$. The reason is that we do not have a simple manner subalgebra structure of the symmetries of (1), as we had for $y^{\prime \prime \prime}=0$.

The Lie algebra of the operators (2) is five-dimensional and has commutator relations given in Table 1.

In order to calculate the adjoint representation, we utilize the Lie series (see Olver [12])

$$
\begin{aligned}
\operatorname{Ad}(\exp (\epsilon X)) Y= & Y-\epsilon[X, Y]+\frac{1}{2 !} \epsilon^{2}[X,[X, Y]] \\
& -\frac{1}{3 !} \epsilon^{3}[X,[X,[X, Y]]]+\cdots
\end{aligned}
$$

together with the commutator table, namely, Table 2 . As an example,

$$
\begin{aligned}
\operatorname{Ad} & \left(\exp \left(\epsilon X_{1}\right)\right) X_{3} \\
& =X_{3}-\epsilon\left[X_{1}, X_{3}\right]+\frac{1}{2 !} \epsilon^{2}\left[X_{1},\left[X_{1}, X_{3}\right]\right]-\cdots \\
& =X_{3}-\epsilon X_{3}+\frac{1}{2 !} \epsilon^{2} X_{3}-\frac{1}{3 !} \epsilon^{3} X_{3}+\cdots \\
& =e^{-\epsilon} X_{3} .
\end{aligned}
$$

In like manner, we obtain the other entries of the adjoint table, and we have the adjoint representation given by Table 2 .

Here, the $(i, j)$ entry represents $\operatorname{Ad}\left(\exp \left(\epsilon X_{i}\right)\right) X_{j}$. For a nonzero vector

$$
X=a_{1} X_{1}+a_{2} X_{2}+\cdots+a_{5} X_{5},
$$

we need to simplify the coefficients $a_{i}$ as far as possible through adjoint maps to $X$. The computations are straightforward, and we find an optimal system of one-dimensional subalgebras spanned by

$$
\begin{gathered}
X_{1}, \\
X_{2}, \\
X_{1} \pm X_{2}, \\
X_{2} \pm X_{4}, \\
a X_{1}+X_{5}, \\
X_{1}+X_{5} \pm X_{3}, \\
-X_{1}+X_{5} \pm X_{4} .
\end{gathered}
$$

The discrete symmetry transformation $y \mapsto-y$ will map $X_{1}-X_{2}$ to $X_{1}+X_{2}$ and that of $x \mapsto-x$ will transform the last entry in (13) to $X_{1}+X_{5} \pm X_{3}$. Also $X_{1}+X_{5}-X_{3}$ will go to $X_{1}+X_{5}+X_{3}$ under $y \mapsto-y$. Therefore the above list (13) is reduced by four.

We now invoke each of the operators of (13) in the classifying relation (8) to systematically work out the symmetry structure of the first integral of (1).

Firstly, we consider $X_{1}$. Since $a_{1}$ is arbitrary, we have

$$
I_{2} \frac{\partial F}{\partial I_{2}}-I_{3} \frac{\partial F}{\partial I_{3}}=0
$$

and hence,

$$
F=F\left(I_{1}, I_{2} I_{3}\right),
$$

which possesses $X_{1}$ as symmetry. After the substitution of (14) into (8) and taking into account (15), we arrive at

$$
\left(-a_{2}+a_{5} I_{1}\right) \frac{\partial F}{\partial I_{1}}+2\left(a_{5} \alpha+a_{3} I_{2}+a_{4} I_{3}\right) \frac{\partial F}{\partial \alpha}=0
$$

where $\alpha=I_{2} I_{3}$. This at once gives $a_{3}=a_{4}=0$.

Note that for $a_{3}, a_{4}$ nonzero, we have $\partial F / \partial \alpha=0$ in which case we further have that $a_{2}=a_{5}=0$. This results in $F=F\left(I_{1}\right)$ which has symmetry generators $X_{1}, X_{3}$, and $X_{4}$ which is the maximal case.

We systematically consider the cases when (16) imply two generators. These arise as follows.

(i) Suppose that $a_{1}, a_{2}$ are arbitrary. Then, (16) gives $\partial F / \partial I_{1}=0$ and

$$
2 \alpha a_{5} \frac{\partial F}{\partial \alpha}=0 .
$$


TABLE 1: The commutation relations for the symmetries of (1).

\begin{tabular}{lccccc}
\hline$\left[X_{i}, X_{j}\right]$ & $X_{1}$ & $X_{2}$ & $X_{3}$ & $X_{4}$ & $X_{5}$ \\
\hline$X_{1}$ & 0 & 0 & $X_{3}$ & $-X_{4}$ & 0 \\
$X_{2}$ & 0 & 0 & 0 & 0 & $X_{2}$ \\
$X_{3}$ & $-X_{3}$ & 0 & 0 & 0 & $X_{3}$ \\
$X_{4}$ & $X_{4}$ & 0 & 0 & 0 & $X_{4}$ \\
$X_{5}$ & 0 & $-X_{2}$ & $-X_{3}$ & 0 & 0 \\
\hline
\end{tabular}

TABLE 2: The adjoint table for the symmetries (2).

\begin{tabular}{cccccc}
\hline Ad & $X_{1}$ & $X_{2}$ & $X_{3}$ & $X_{4}$ & $X_{5}$ \\
\hline$X_{1}$ & $X_{1}$ & $X_{2}$ & $e^{-\epsilon} X_{3}$ & $e^{\epsilon} X_{4}$ & $X_{5}$ \\
$X_{2}$ & $X_{1}$ & $X_{2}$ & $X_{3}$ & $X_{4}$ & $X_{5}-\epsilon X_{2}$ \\
$X_{3}$ & $X_{1}+\epsilon X_{3}$ & $X_{2}$ & $X_{3}$ & $X_{4}$ & $X_{5}-\epsilon X_{3}$ \\
$X_{4}$ & $X_{1}-\epsilon X_{4}$ & $X_{2}$ & $X_{3}$ & $X_{4}$ & $X_{5}-\epsilon X_{4}$ \\
$X_{5}$ & $X_{1}$ & $e^{\epsilon} X_{2}$ & $e^{\epsilon} X_{3}$ & $e^{\epsilon} X_{4}$ & $X_{5}$ \\
\hline
\end{tabular}

For $F$, not a constant, we must have that $a_{5}=0$, and we get

$$
F=F\left(I_{2} I_{3}\right)
$$

which has $X_{1}$ and $X_{2}$ as symmetries.

(ii) Suppose that $a_{1}, a_{5}$ are arbitrary. Then, (16) implies that

$$
I_{1} \frac{\partial F}{\partial I_{1}}+2 \alpha \frac{\partial F}{\partial I_{1}}=0
$$

from which we arrive at

$$
F=F\left(I_{1}\left(I_{2} I_{3}\right)^{-1 / 2}\right)
$$

This integral (20) has $X_{1}$ and $X_{5}$ as symmetry generators.

We do not obtain any further three symmetry cases from (16) apart from the earlier for $I_{1}$ as it gives $F$ a constant and hence no integral.

Next we focus on $X_{2}$. The use of the classifying relation (8) gives rise to

$$
\left[\left(a_{1}+a_{5}\right) I_{2}+2 a_{4}\right] \frac{\partial F}{\partial I_{2}}+\left[\left(a_{5}-a_{1}\right) I_{3}+2 a_{3}\right] \frac{\partial F}{\partial I_{3}}=0
$$

and therefore,

$$
F=F\left(I_{2}, I_{3}\right)
$$

admits $X_{2}$. In a similar manner as for $X_{1}$ we have the following cases.

(i) If $a_{1}, a_{2}$ are arbitrary, then we obtain $F$ as in (15).

(ii) If $a_{2}, a_{3}$ are arbitrary, then we have $X_{2}, X_{3}, X_{1}-X_{5}$, and $F=F\left(I_{2}\right)$.

(iii) If $a_{2}, a_{4}$ are arbitrary, then we have $X_{2}, X_{4}, X_{1}+X_{5}$, and $F=F\left(I_{3}\right)$. (iv) If $a_{2}, a_{5}$ are arbitrary, then $X_{2}, X_{5}$ result in $F=$ $F\left(I_{3} / I_{2}\right)$.

We do not get any three symmetry case here.

The pattern is now clear. Instead of going through each of the remaining cases which are quite tedious albeit straightforward, we present our findings in a table. For completeness, this table also includes the cases $X_{1}$ and $X_{2}$ together with the corresponding first integrals (see Tables 3 and 4 ).

Finally, we look at the three symmetry cases.

For $I_{1}$, there are three symmetries

$$
\begin{aligned}
& X_{1}=\exp x \frac{\partial}{\partial y} \\
& X_{2}=\exp (-x) \frac{\partial}{\partial y} \\
& X_{3}=\frac{\partial}{\partial x}
\end{aligned}
$$

which have nonzero commutation relations

$$
\left[X_{1}, X_{3}\right]=-X_{1}, \quad\left[X_{2}, X_{3}\right]=X_{2} .
$$

The Lie algebra is $L_{3 ; 4}$. In the case of the first integral $I_{2}$, the symmetries are

$$
\begin{aligned}
& X_{1}=\exp x \frac{\partial}{\partial y}, \\
& X_{2}=\frac{\partial}{\partial y}, \\
& X_{3}=\frac{1}{2} y \frac{\partial}{\partial y}-\frac{1}{2} \frac{\partial}{\partial x},
\end{aligned}
$$

which have nonzero Lie brackets

$$
\left[X_{1}, X_{3}\right]=X_{1}, \quad\left[X_{2}, X_{3}\right]=\frac{1}{2} X_{2}
$$

and constitute the Lie algebra $L_{3 ; 5}, a=1 / 2$. The Lie algebra of the symmetries of $I_{3}$ is isomorphic to that of $I_{2}$ by means of the discrete transformation $\bar{x}=-x$.

Thus, there are two Lie algebras of dimension three, namely, $L_{3 ; 4}$ and $L_{3 ; 5}, a=1 / 2$. There are no four symmetry cases. Therefore, we have the following result.

Theorem 1. The maximal dimension of the Lie algebra admitted by a first integral of $y^{\prime \prime \prime}-y^{\prime}=0$ or a third-order ODE linearizable by point transformation to this linear ODE is three. The maximal Lie algebras are $L_{3 ; 4}$ and $L_{3 ; 5}, a=1 / 2$.

The proof follows easily from the preceding discussion.

We also have the following counting theorem.

Theorem 2. The Lie algebra admitted by a first integral of $y^{\prime \prime \prime}-y^{\prime}=0$ or a third-order ODE linearizable by point transformation to this linear $O D E$ is $0,1,2$, or 3.

The proof follows from (9), Tables 3 and 4 and Theorem 1. 
TABLE 3: One symmetry cases and the integrals of (1).

\begin{tabular}{lc}
\hline One symmetry & First integral \\
\hline$X_{1}$ & $F=F\left(I_{1}, I_{2} I_{3}\right)$ \\
$X_{2}$ & $F=F\left(I_{2}, I_{3}\right)$ \\
$X_{1}+X_{2}$ & $F=F\left(I_{2} I_{3}, I_{2} \exp \left(I_{1}\right)\right)$ \\
$X_{2} \pm X_{4}$ & $F=F\left(I_{2} \pm I_{1}, I_{3}\right)$ \\
$X_{5}$ & $F=F\left(I_{3} /\left(I_{2} \pm I_{1}\right)\right)$ \\
$X_{5}+a X_{1}, a \neq 0$ & $F=F\left(I_{2} I_{1}^{-1-a}, I_{3} I_{1}^{a-1}\right)$ \\
$X_{1}+X_{5}+X_{3}$ & $F=F\left(I_{2} I_{1}^{-2}, I_{3}-\ln I_{2}\right)$ \\
\hline
\end{tabular}

\section{Algebraic Properties of the Integrals of}

$$
y^{\prime \prime \prime}+f y^{\prime \prime}-y^{\prime}-f y=0
$$

We consider the representative third-order ODE

$$
y^{\prime \prime \prime}+f(x) y^{\prime \prime}-y^{\prime}-f(x) y=0,
$$

where $f$ is an arbitrary function of $x$. This equation possesses four symmetries

$$
\begin{aligned}
& X_{1}=e^{x} \frac{\partial}{\partial y}, \\
& X_{2}=e^{-x} \frac{\partial}{\partial y}, \\
& X_{3}=\alpha(x) \frac{\partial}{\partial y}, \\
& X_{4}=y \frac{\partial}{\partial y},
\end{aligned}
$$

where again we commenced with the three solution symmetries and then the homogeneity symmetry. Here, $\alpha=$ $(1 / 2) e^{x} \int e^{\left(-x-\int f(x) d x\right)} d x-(1 / 2) e^{-x} \int e^{\left(x-\int f(x) d x\right)} d x$ is a solution of (27). The third-order equation (27) has the three functionally independent first integrals

$$
\begin{aligned}
& I_{1}=\left(y^{\prime \prime}-y\right) e^{\int f(x) d x}, \\
& I_{2}=y e^{-x}+y^{\prime} e^{-x}-\left[\int e^{\left(-x-\int f(x) d x\right)} d x\right]\left(y^{\prime \prime}-y\right) e^{\int f(x) d x}, \\
& I_{3}=y e^{x}-y^{\prime} e^{x}+\left[\int e^{\left(x-\int f(x) d x\right)} d x\right]\left(y^{\prime \prime}-y\right) e^{\int f(x) d x} .
\end{aligned}
$$

The first in this list is the simplest, followed by the other two for which the order does not matter.

3.1. Classifying Relation for the Symmetries of $y^{\prime \prime \prime}+f y^{\prime \prime}-$ $y^{\prime}-f y=0$. Let $F$ be an arbitrary function of $I_{1}, I_{2}$, and $I_{3}$; namely, $F=F\left(I_{1}, I_{2}, I_{3}\right)$. The symmetry of this general function of the first integrals is

$$
X^{[2]} F=X^{[2]} I_{1} \frac{\partial F}{\partial I_{1}}+X^{[2]} I_{2} \frac{\partial F}{\partial I_{2}}+X^{[2]} I_{3} \frac{\partial F}{\partial I_{3}}=0,
$$

TABle 4: Two symmetry cases and the integrals of (1).

\begin{tabular}{lc}
\hline Two symmetries & First integral \\
\hline$X_{1}, X_{2}$ & $F=F\left(I_{2} I_{3}\right)$ \\
$X_{1}, X_{5}$ & $F=F\left(I_{1}\left(I_{2} I_{3}\right)^{-1 / 2}\right)$ \\
$X_{2}, X_{5}$ & $F=F\left(I_{3} / I_{2}\right)$ \\
$X_{2} \pm X_{4}, X_{5}$ & $F=F\left(I_{3} /\left(I_{2} \pm I_{1}\right)\right)$ \\
$X_{3}, X_{5}$ & $F=F\left(I_{2} / I_{1}\right)$ \\
$X_{5}+a X_{1}, X_{2}, a \neq 0$ & $F=F\left(I_{3} I_{2}^{a-1 / a+1}\right)$ \\
$X_{5}+a X_{1}, X_{3}$ & $F=F\left(I_{2} I_{1}^{-1-a}\right)$ \\
$X_{2}, X_{1}+X_{5}+X_{3}$ & $F=F\left(I_{3}-\ln I_{2}\right)$ \\
$X_{4}, X_{1}+X_{5}+X_{3}$ & $F=F\left(I_{3}-\ln I_{1}^{2}\right)$ \\
\hline
\end{tabular}

where

$$
\begin{aligned}
X^{[2]} I_{1}= & {\left[\xi \frac{\partial}{\partial x}+\eta \frac{\partial}{\partial y}+\zeta_{x} \frac{\partial}{\partial y^{\prime}}+\zeta_{x x} \frac{\partial}{\partial y^{\prime \prime}}\right] } \\
& \times\left(y^{\prime \prime}-y\right) e^{\int f(x) d x} \\
= & \xi\left[\left(y^{\prime \prime}-y\right) e^{\int f(x) d x} f(x)\right]-\eta e^{\int f(x) d x} \\
& +\zeta_{x x} e^{\int f(x) d x} \\
X^{[2]} I_{2}= & \left.\xi \frac{\partial}{\partial x}+\eta \frac{\partial}{\partial y}+\zeta_{x} \frac{\partial}{\partial y^{\prime}}+\zeta_{x x} \frac{\partial}{\partial y^{\prime \prime}}\right] \\
\times & \left(y e^{-x}+y^{\prime} e^{-x}\right. \\
& \left.-\left[\int e^{\left(-x-\int f(x) d x\right)} d x\right]\left(y^{\prime \prime}-y\right) e^{\int f(x) d x}\right) \\
= & -\left(y+y^{\prime}\right) e^{-x}-\left(\int e^{\left(-x-\int f(x) d x\right)} d x\right) \\
& \times\left(y^{\prime \prime}-y\right) e^{\int f(x) d x} f(x) \\
& \left.-\left(y^{\prime \prime}-y\right) e^{\int f(x) d x} e^{\left(-x-\int f(x) d x\right)}\right] \xi \\
+ & {\left[e^{-x}+\left(e^{\int f(x) d x}\right)\left(\int e^{\left(-x-\int f(x) d x\right)} d x\right)\right] \eta } \\
+ & +\left(e^{-x} \zeta_{x}-\left[\left(\int e^{\left(-x-\int f(x) d x\right)} d x\right) e^{\int f(x) d x}\right] \zeta_{x x}\right. \\
X^{[2]} I_{3}= & {\left[\xi \frac{\partial}{\partial x}+\eta \frac{\partial}{\partial y}+\zeta_{x} \frac{\partial}{\partial y^{\prime}}+\zeta_{x x} \frac{\partial}{\partial y^{\prime \prime}}\right] } \\
\times & \left(y e^{x}-y^{\prime} e^{x}\right. \\
& \left.+\left[\int e^{\left(x-\int f(x) d x\right.} e^{\left(x-\int f(x) d x\right)} d x\right]\left(y^{\prime \prime}-y\right) e^{\int f(x) d x}\right) \\
& {\left[\left(y-y^{\prime}\right) e^{x}+\left(\int e^{\left(x-\int f(x) d x\right)} d x\right)\right.} \\
& +y) e^{\int f(x) d x} f(x) \\
& +(x) \\
&
\end{aligned}
$$




$$
\begin{aligned}
& +\left[e^{x}-\left(e^{\int f(x) d x}\right)\left(\int e^{\left(x-\int f(x) d x\right)} d x\right)\right] \eta-e^{x} \zeta_{x} \\
& +\left[\left(\int e^{\left(x-\int f(x) d x\right)} d x\right) e^{\int f(x) d x}\right] \zeta_{x x} .
\end{aligned}
$$

Now $\xi, \eta, \zeta_{x}$, and $\zeta_{x x}$ are

$$
\begin{gathered}
\xi=0, \\
\eta=e^{x} a_{1}+e^{-x} a_{2}+\alpha(x) a_{3}+y a_{4}, \\
\zeta_{x}=e^{x} a_{1}-e^{-x} a_{2}+\alpha^{\prime}(x) a_{3}+y^{\prime} a_{4}, \\
\zeta_{x x}=e^{x} a_{1}+e^{-x} a_{2}+\alpha^{\prime \prime}(x) a_{3}+y^{\prime \prime} a_{4} .
\end{gathered}
$$

These are the coefficients functions of $X^{[2]}$ which are obtained by setting

$$
X^{[2]}=\sum_{i=1}^{4} a_{i} X_{i}^{[2]},
$$

where $X_{i}$ s are the symmetry generators as given in (28) and the $a_{i}$ s are constants. The reason for taking a linear combination mentioned earlier is that the symmetries of the first integrals are always the symmetries of the equation (see [11] for a general result).

After insertion of the values of $X^{[2]} I_{1}, X^{[2]} I_{2}$, and $X^{[2]} I_{3}$ as in (31), with $\xi, \eta, \zeta_{x}$, and $\zeta_{x x}$ as in (32), and first integrals $I_{1}=$ $\left(y^{\prime \prime}-y\right) e^{\int f(x) d x}, I_{2}=y e^{-x}+y^{\prime} e^{-x}-\left[\int e^{\left(-x-\int f(x) d x\right)} d x\right]\left(y^{\prime \prime}-\right.$ y) $e^{\int f(x) d x}$, and $I_{3}=y e^{x}-y^{\prime} e^{x}+\left[\int e^{\left(x-\int f(x) d x\right)} d x\right]\left(y^{\prime \prime}-\right.$ $y) e^{\int f(x) d x}$ as well as use of

$$
\begin{gathered}
\alpha^{\prime \prime}-\alpha=e^{-\int f(x) d x}, \\
\alpha^{\prime}+\alpha=e^{x} \int e^{\left(-x-\int f(x) d x\right)} d x, \\
\alpha-\alpha^{\prime}=-e^{-x} \int e^{\left(x-\int f(x) d x\right)} d x, \\
y^{\prime \prime}-y=I_{1} e^{-\int f(x) d x}, \\
\left(y^{\prime}+y\right) e^{-x}=I_{2}+I_{1} \int e^{\left(-x-\int f(x) d x\right)} d x, \\
\left(y-y^{\prime}\right) e^{x}=I_{3}-I_{1} \int e^{\left(x-\int f(x) d x\right)} d x
\end{gathered}
$$

in (30), we eventually find the classifying relation

$$
\begin{gathered}
\left(a_{3}+I_{1} a_{4}\right) \frac{\partial F}{\partial I_{1}}+\left(2 a_{1}+I_{2} a_{4}\right) \frac{\partial F}{\partial I_{2}} \\
+\left(2 a_{2}+I_{3} a_{4}\right) \frac{\partial F}{\partial I_{3}}=0 .
\end{gathered}
$$

The relation (35) provides the relationship between the symmetries and first integrals of the third-order equation (27). We utilize this to classify the first integrals in terms of their symmetries.
3.2. Symmetry Structure of the First Integrals of $y^{\prime \prime \prime}+f y^{\prime \prime}-$ $y^{\prime}-f y=0$. We use the relation (35) to systematically study the relationship between the symmetries and first integrals of (27).

We quickly note that if $F$ is arbitrary, then (35) implies

$$
\begin{aligned}
& a_{3}+I_{1} a_{4}=0, \\
& 2 a_{1}+I_{2} a_{4}=0, \\
& 2 a_{4}+I_{3} a_{4}=0,
\end{aligned}
$$

which in turn give the result that the $a$ 's are zero. Thus, there results no symmetry for this case.

As in the previous section on the constant coefficient ODE, we obtain the optimal system of one-dimensional subalgebras of the four-dimensional algebra symmetry algebra of our ODE spanned by (28).

The Lie algebra of the symmetries (28) is represented by Table 5.

By use of Table 5, we can construct the adjoint representation which we present in Table 6.

We then obtain an optimal system of one-dimensional subalgebras spanned by

$$
\begin{gathered}
X_{3}, \\
X_{4}, \\
X_{2}+a X_{3}, \\
X_{1}+a X_{2}+b X_{3} .
\end{gathered}
$$

For each of these operators, we are systematically able to compute the corresponding first integrals by using the classifying relation (35).

In Tables 7 and 8 we tabulate the symmetries and the corresponding first integrals.

It follows that there are no three symmetry cases. Moreover, we note that the maximal case of symmetries of the first integrals for (27) is two and these are listed in Table 8.

We therefore have the following result.

Theorem 3. The Lie algebra admitted by a first integral of $y^{\prime \prime \prime}+$ $f(x) y^{\prime \prime}-y^{\prime}-f(x) y=0$ or a third-order ODE linearizable by point transformation to this linear ODE is 0,1 , or 2.

The proof follows from (36) and Tables 7 and 8.

\section{Further Considerations: Symmetries of First Integrals of Submaximal Higher-Order ODEs}

We know that one cannot generate the full Lie algebra of any scalar first-order ODE via the algebras of any of its integrals [10]. Also for scalar linear second-order ODEs, it has been shown in [6] that the full Lie algebra of $y^{\prime \prime}=0$ which represents any linear or linearizable second-order ODE can be generated by three isomorphic triplets of three-dimensional algebras of the basic integrals and one of their quotient which have the interesting property that the algebras are isomorphic to each other. In our recent work [10], we have pointed out 
TABLE 5: The commutation relations for the symmetries of (27).

\begin{tabular}{lcccc}
\hline$\left[X_{i}, X_{j}\right]$ & $X_{1}$ & $X_{2}$ & $X_{3}$ & $X_{4}$ \\
\hline$X_{1}$ & 0 & 0 & 0 & $X_{1}$ \\
$X_{2}$ & 0 & 0 & 0 & $X_{2}$ \\
$X_{3}$ & 0 & 0 & 0 & $X_{3}$ \\
$X_{4}$ & $-X_{1}$ & $-X_{2}$ & $-X_{3}$ & 0 \\
\hline
\end{tabular}

TABLE 6: The adjoint table for the symmetries (28).

\begin{tabular}{ccccc}
\hline Ad & $X_{1}$ & $X_{2}$ & $X_{3}$ & $X_{4}$ \\
\hline$X_{1}$ & $X_{1}$ & $X_{2}$ & $X_{3}$ & $X_{4}-\epsilon X_{1}$ \\
$X_{2}$ & $X_{1}$ & $X_{2}$ & $X_{3}$ & $X_{4}-\epsilon X_{2}$ \\
$X_{3}$ & $X_{1}$ & $X_{2}$ & $X_{3}$ & $X_{4}-\epsilon X_{3}$ \\
$X_{4}$ & $e^{\epsilon} X_{1}$ & $e^{\epsilon} X_{2}$ & $e^{\epsilon} X_{3}$ & $X_{4}$ \\
\hline
\end{tabular}

that the full Lie algebra of the simplest third-order equation $y^{\prime \prime \prime}=0$ is generated by the point symmetries of only two of the basic integrals $I_{1}$ and $I_{3}$ from the three

$$
\begin{aligned}
& I_{1}=y^{\prime \prime}, \\
& I_{2}=x y^{\prime \prime}-y^{\prime}, \\
& I_{3}=\frac{1}{2} x^{2} y^{\prime \prime}-x y^{\prime}+y .
\end{aligned}
$$

This is indeed very different to what happens to the classes $y^{\prime}=0$ and $y^{\prime \prime}=0$. One has that the seven symmetries of our the simplest third-order ODE are generated by four symmetries of $I_{1}$ together with three symmetries of $I_{3}$. In the case of higher-order ODEs of maximal symmetry, it was shown in [10] that similar properties persist. That is, the full Lie algebra of $y^{(n)}=0, n \geq 3$ is generated by two subalgebras, namely the $n+1$-dimensional algebra of the integral $I_{1}=$ $y^{(n-1)}$ and the three-dimensional subalgebra of the integral $I_{n}=\sum_{i=1}^{n}\left((-1)^{i-1} /(n-i) !\right) x^{n-i} y^{(n-i)}$.

What occurs to higher-order ODEs of submaximal symmetry? We discuss this in the following.

Consider the $n$ th-order ODE of submaximal symmetry

$$
y^{(n)}-y^{(n-2)}=0, \quad n \geq 3 .
$$

This ODE (39) can be taken as a representative of higherorder ODEs which has $n+2$-point symmetries. We have chosen this in a way that reduces to the third-order case focused on earlier. The $n$ first integrals of (39) have the same pattern as for the third-order case and are thus easily constructible, and we focus on the first and second which are

$$
\begin{aligned}
& I_{1}=y^{(n-1)}-y^{(n-3)}, \\
& I_{2}=e^{x}\left(y^{(n-1)}-y^{(n-2)}\right) .
\end{aligned}
$$

The first integral (40) has $n$ point symmetries

$$
\begin{gathered}
X_{1}=e^{x} \frac{\partial}{\partial x}, \quad X_{2}=e^{-x} \frac{\partial}{\partial y}, \quad X_{3}=\frac{\partial}{\partial x}, \\
X_{i}=x^{i-4} \frac{\partial}{\partial y}, \quad i=4, \ldots, n .
\end{gathered}
$$

TABLE 7: One symmetry cases and the integrals of (27).

\begin{tabular}{lc}
\hline One symmetry & First integral \\
\hline$X_{3}$ & $F=F\left(I_{2}, I_{3}\right)$ \\
$X_{4}$ & $F=F\left(I_{2} / I_{1}, I_{3} / I_{2}\right)$ \\
$X_{2}+a X_{3}$ & $F=F\left(I_{2} I_{3}, I_{2} \exp \left(I_{1}\right)\right)$ \\
$X_{2} \pm X_{4}$ & $F=F\left(I_{1}-(1 / 2) a I_{3}, I_{2}\right)$ \\
$X_{1}+a X_{2}$ & $F=F\left(I_{3}-a I_{2}, I_{1}\right)$ \\
$X_{1}+a X_{2}$ & $F=F\left(I_{1}-(1 / 2) a I_{2}, I_{3}\right)$ \\
$X_{1}+a X_{2}+b X_{3}, a, b \neq 0$ & $F=F\left(b I_{3}-2 a I_{1}, I_{2}\right)$ \\
\hline
\end{tabular}

TABLE 8: Two symmetry cases and the integrals of (27).

\begin{tabular}{lc}
\hline Two symmetries & First integral \\
\hline$X_{2}, X_{3}$ & $F=F\left(I_{2}\right)$ \\
$X_{3}, X_{4}$ & $F=F\left(I_{3} / I_{2}\right)$ \\
$X_{1}, X_{2}+a X_{3}$ & $F=F\left(I_{1}-(1 / 2) a I_{3}\right)$ \\
$X_{2}+a X_{3}, X_{4}$ & $F=F\left(I_{2} /\left(I_{1}-(1 / 2) a I_{3}\right)\right)$ \\
$X_{1}+a X_{2}, X_{3}$ & $F=F\left(I_{3}-a I_{2}\right)$ \\
$X_{1}+a X_{2}, X_{4}$ & $F=F\left(I_{3}-a I_{2} / I_{2}\right)$ \\
$X_{1}+a X_{3}, X_{2}, a \neq 0$ & $F=F\left(I_{1}-(1 / 2) a I_{2}\right)$ \\
$X_{1}+a X_{3}, X_{4}, a \neq 0$ & $F=F\left(I_{1}-(1 / 2) a I_{2} / I_{3}\right)$ \\
$X_{2}+a X_{3}, X_{1}, a \neq 0$ & $F=F\left(I_{1}-(1 / 2) a I_{3}\right)$ \\
$X_{1}+a X_{2}+b X_{3}, X_{4}, a, b \neq 0$ & $F=F\left(b I_{3}-2 a I_{1} / I_{2}\right)$ \\
\hline
\end{tabular}

This forms an $n$-dimensional subalgebra of the symmetry algebra of (39). The nonzero commutation relations are

$$
\begin{aligned}
& {\left[X_{1}, X_{3}\right]=-X_{1}, \quad\left[X_{2}, X_{3}\right]=X_{2}} \\
& {\left[X_{3}, X_{i}\right]=(i-4) X_{i-1}, \quad i=4, \ldots, n}
\end{aligned}
$$

The first integral (41) has point symmetries

$$
\begin{gathered}
Y_{1}=e^{x} \frac{\partial}{\partial y} \\
Y_{i}=x^{i-2} \frac{\partial}{\partial y}, \quad i=2, \ldots, n-1, \\
Y_{n}=y \frac{\partial}{\partial y}-\frac{\partial}{\partial x} .
\end{gathered}
$$

These generators have nonzero commutation relations

$$
\begin{gathered}
{\left[Y_{1}, Y_{n}\right]=2 Y_{1},} \\
{\left[Y_{i}, Y_{n}\right]=Y_{i}+(i-2) Y_{i-1}, \quad i=2, \ldots, n-1 .}
\end{gathered}
$$

We see that these two sets of symmetries (42) and (44) are easy to deduce as it is clear that (42) form symmetries of (40) since they are translation in $x$ and solution symmetries with maximum degree power $x^{(n-4)}$. Also for $n=3$, they reduce to the third-order case of the previous section. The full Lie algebra of (39) is generated from the $n$ symmetries of (42) and two symmetries of (44), namely, $Y_{n-1}$ and $Y_{n}$ of (44). However, the latter does not close due to the commutation relations (45). However, if we exclude $Y_{1}$, then $\left\langle Y_{2}, \ldots, Y_{n}\right\rangle$ 
does span an $(n-1)$-dimensional algebra. Alternatively, a simpler way to generate the full algebra of (39) is to utilize the symmetries (44) together with the two symmetries $X_{2}$ and $X_{3}$ of (42).

We therefore have the theorem the proof of which follows from the above discussion.

Theorem 4. The full Lie algebra of the linear nth-order ODE $y^{(n)}-y^{(n-1)}=0, n \geq 3$, which is $n+2$ dimensional, is generated by two subalgebras, namely, the n-dimensional algebra $\left\langle Y_{j}: j=1, \ldots, n\right\rangle$ of $I_{2}=e^{x}\left(y^{(n-1)}-y^{(n-2)}\right)$ and the two-dimensional subalgebra $\left\langle X_{2}, X_{3}\right\rangle$ of $I_{1}=y^{(n-1)}-y^{(n-3)}$.

We now study the generation of the full algebra of a representative $n$ th-order, $n \geq 3$ of submaximal symmetries $n+1$. A natural extension of the third-order ODE (27) is

$$
y^{(n)}-y^{(n-2)}+f(x)\left(y^{(n-1)}-y^{(n-3)}\right)=0, \quad n \geq 3,
$$

where $f(x)$ is an arbitrary function of $x$. Following the pattern of the integrals in (29), we can write the corresponding three out of $n$ immediately. They are

$$
\begin{aligned}
I_{1}= & \left(y^{(n-1)}-y^{(n-3)}\right) e^{\int f(x) d x}, \\
I_{2}= & e^{-x}\left(y^{(n-2)}+y^{(n-3)}\right)-\left[\int e^{\left(-x-\int f(x) d x\right)} d x\right] \\
& \times\left(y^{(n-1)}-y^{(n-3)}\right) e^{\int f(x) d x}, \\
I_{3}= & e^{x}\left(y^{(n-3)}-y^{(n-2)}\right)+\left[\int e^{\left(x-\int f(x) d x\right)} d x\right] \\
& \times\left(y^{(n-1)}-y^{(n-3)}\right) e^{\int f(x) d x} .
\end{aligned}
$$

We show that the symmetries of these integrals are sufficient to generate the full algebra. From Table 8 in the previous section, we notice that $X_{1}$ and $X_{2}$ of (28) are symmetries of the integral $I_{1}$ in (29). Further we note that $X_{3}$ and $X_{4}$ of (28) are symmetries of the quotient integral $I_{3} / I_{2}$. In a similar fashion, we have these algebraic properties persisting for the linear higher-order equation (46). Equation (46) has the $n+1$ point symmetries

$$
\begin{gathered}
X_{1}=e^{x} \frac{\partial}{\partial y}, \quad X_{2}=e^{-x} \frac{\partial}{\partial y} \\
X_{i}=x^{i-3} \frac{\partial}{\partial y}, \quad i=3, \ldots, n-1, \\
X_{n}=\alpha(x) \frac{\partial}{\partial y}, \\
X_{n+1}=y \frac{\partial}{\partial y},
\end{gathered}
$$

where $\alpha$ is a solution to (46) and satisfies similar properties to that of the corresponding linear third-order equation; namely,

$$
\begin{gathered}
\alpha^{(n-1)}-\alpha^{(n-3)}=e^{-\int f(x) d x} \\
\alpha^{(n-1)}-\alpha^{(n-2)}=-e^{x} \int e^{x-\int f(x) d x} d x+e^{-\int f(x) d x}, \\
\alpha^{(n-2)}+\alpha^{(n-3)}=e^{x} \int e^{-x-\int f(x) d x} d x .
\end{gathered}
$$

It is evident that the first $n$ are solution symmetries and the $(n+1)$ th is the homogeneity symmetry which are straightforward to observe. The first integral $I_{1}$ in (47) has the $n-1$ symmetries $X_{1}, \ldots, X_{n-1}$ which is clear. The algebra constituted is Abelian. This fact can also be seen for $I_{1}$ of (29). Now, we analyze what occurs for the quotient integral $I_{3} / I_{2}$ of (47). It is noticed that the homogeneity symmetry $X_{n+1}$ is a symmetry of $I_{3} / I_{2}$, as if we replace $y$ by $\gamma y$ in the quotient; it is left invariant. Moreover, for $X_{n}$ we have the invariance condition

$$
\begin{gathered}
X_{n}^{(n-1)}\left(\frac{I_{3}}{I_{2}}\right) \\
=\frac{1}{I_{2}}\left\{\left[e^{x}\left(\alpha^{(n-3)}-\alpha^{(n-2)}\right)\right.\right. \\
\left.+\int e^{x-\int f(x) d x} e^{\int f(x) d x} d x\left(\alpha^{(n-1)}-\alpha^{(n-3)}\right)\right] \\
-\frac{I_{3}}{I_{2}}\left[e^{-x}\left(\alpha^{(n-2)}+\alpha^{(n-3)}\right)\right. \\
-\int e^{-x-\int f(x) d x} e^{\int f(x) d x} d x \\
\left.\left.\times\left(\alpha^{(n-1)}-\alpha^{(n-3)}\right)\right]\right\}=0
\end{gathered}
$$

The terms in the square brackets vanish due to the relations in (49). Thus, $X_{n}$ is a symmetry of this quotient integral. In view of the previous, we have the following theorem.

Theorem 5. The full Lie algebra of the linear nth-order ODE $y^{(n)}-y^{(n-2)}+f(x)\left(y^{(n-1)}-y^{(n-3)}\right)=0, n \geq 3$, which is $n+1$ dimensional, is generated by two subalgebras, namely, the ( $n-$ 1)-dimensional algebra $\left\langle X_{j}: j=1, \ldots, n-1\right\rangle$ of $I_{1}$ as given in (47) and the two-dimensional subalgebra $\left\langle X_{n}, X_{n+1}\right\rangle$ of $I_{3} / I_{2}$ as in (47).

Hence, the manner in which the full Lie algebra is generated for the ODEs $y^{\prime \prime}=0[6], y^{(n)}=0, n \geq 3[10]$, and two submaximal linear cases investigated in the foregoing is quite interesting. This also conforms with the properties of their symmetry algebra which are different (see, e.g., [5]).

\section{Conclusion}

The algebraic properties of the first integrals of the 8 symmetries or maximal class were pursued in [6] in which it was 
shown that the algebra $\mathrm{sl}(3, R)$ of the linearizable equations can be generated by three isomorphic triplets of threedimensional algebras. Then, in [7] the authors considered the symmetry properties of the basic first integrals of scalar linear third-order ODEs for which the symmetry structure has been investigated before (see, e.g., the review [5]). In a recent paper we performed a complete study of the symmetry structure of first integrals of the free particle or linearizable secondorder ODEs. We showed in our work [9] that the first integrals have rich symmetry algebras. We found that they have 0,1 , 2 , or 3 dimensional algebras and that the maximal case is unique with algebra $L_{3,5}^{I}$. Motivated by this and recent works [6-8], we performed in [10] a symmetry classification of the first integrals of the maximal class of linear third-order ODEs represented by $y^{\prime \prime \prime}=0$. Many interesting properties came to light. It was shown in [10] that the symmetry structure of the first integrals is also rich, and there exit the $0,1,2$, and 3 symmetry cases. In the case of the maximal algebra of the integrals which is 3 here, we showed that similar to the free particle case, it is unique. We also proved that the full Lie algebra of the equation for linear third and higher order can be generated by just two basic integrals. This result differs from what happens to the free particle or even first order equations [9].

In this work, we investigated the symmetry properties of the first integrals of scalar linearizable third-order ODEs of submaximal classes, namely, the 4 and 5 symmetry classes. Here we obtained the result that there can be the 0,1 , or 2 symmetry cases for the 4 symmetry class and $0,1,2$, or 3 symmetry cases for the 5 symmetry class. Also we noted that the maximal cases are not unique as for the free particle or simplest third-order equations. We further studied the generation of the full Lie algebras of the submaximal classes of linear higher-order ODEs and have shown how these are generated by subalgebras of certain basic integrals and a quotient of two integrals.

Further work could be done to study submaximal classes of higher order ODEs for the symmetry properties of their first integrals.

\section{Conflict of Interests}

The authors declare that there is no conflict of interests in the publication of this work.

\section{Acknowledgments}

K. S. Mahomed thanks the University of the Witwatersrand as well as the NRF of South Africa for financial support. E. Momoniat is grateful to the NRF for a research grant.

\section{References}

[1] S. Lie, "Klassifikation und Integration von gewöhnlichen differentialgleichungen zwischen $x, y$, die eine Gruppe von Transformationen gestatten," Archiv der Mathematik, vol. 8, no. 9, 1883.

[2] A. M. Tresse, "Sur les invariants différentiels des groupes continus de transformations," Acta Mathematica, vol. 18, no. 1, pp. $1-88,1894$.
[3] F. M. Mahomed, A. H. Kara, and P. G. L. Leach, "Lie and Noether counting theorems for one-dimensional systems," Journal of Mathematical Analysis and Applications, vol. 178, no. 1, pp. 116129, 1993.

[4] F. M. Mahomed and P. G. L. Leach, "Symmetry Lie algebras of $n$th order ordinary differential equations," Journal of Mathematical Analysis and Applications, vol. 151, no. 1, pp. 80-107, 1990.

[5] N. H. Ibragimov, CRC Handbook of Lie Group Analysis of Differential Equations, vol. 3, CRC Press, Boca Raton, Mass, USA, 1996.

[6] P. G. L. Leach and F. M. Mahomed, "Maximal subalgebra associated with a first integral of a system possessing $\operatorname{sl}(3, \mathbf{R})$ algebra," Journal of Mathematical Physics, vol. 29, no. 8, pp. 18071813, 1988.

[7] K. S. Govinder and P. G. L. Leach, "The algebraic structure of the first integrals of third-order linear equations," Journal of Mathematical Analysis and Applications, vol. 193, no. 1, pp. 114133, 1995.

[8] G. P. Flessas, K. S. Govinder, and P. G. L. Leach, "Characterisation of the algebraic properties of first integrals of scalar ordinary differential equations of maximal symmetry," Journal of Mathematical Analysis and Applications, vol. 212, no. 2, pp. 349-374, 1997.

[9] K. S. Mahomed and E. Momoniat, "Symmetry classification of first integrals for scalar linearizable second-order ODEs," Journal of Applied Mathematics, vol. 2012, Article ID 847086, 14 pages, 2012.

[10] K. S. Mahomed and E. Momoniat, "Algebraic properties of first integrals for scalar linear third-order ODEs of maximal symmetry," Abstract and Applied Analysis, vol. 2013, Article ID 530365, 8 pages, 2013.

[11] A. H. Kara and F. M. Mahomed, "A basis of conservation laws for partial differential equations," Journal of Nonlinear Mathematical Physics, vol. 9, supplement 2, pp. 60-72, 2002.

[12] P. J. Olver, Applications of Lie Groups to Differential Equations, Springer, New York, NY, USA, 1986. 


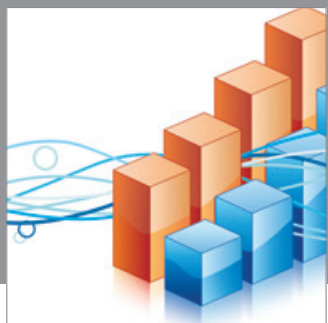

Advances in

Operations Research

mansans

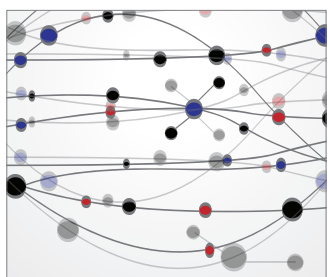

The Scientific World Journal
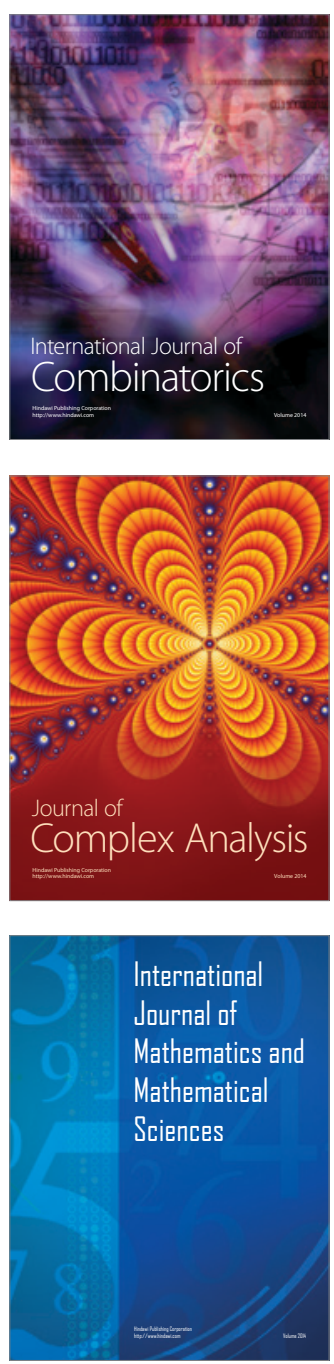
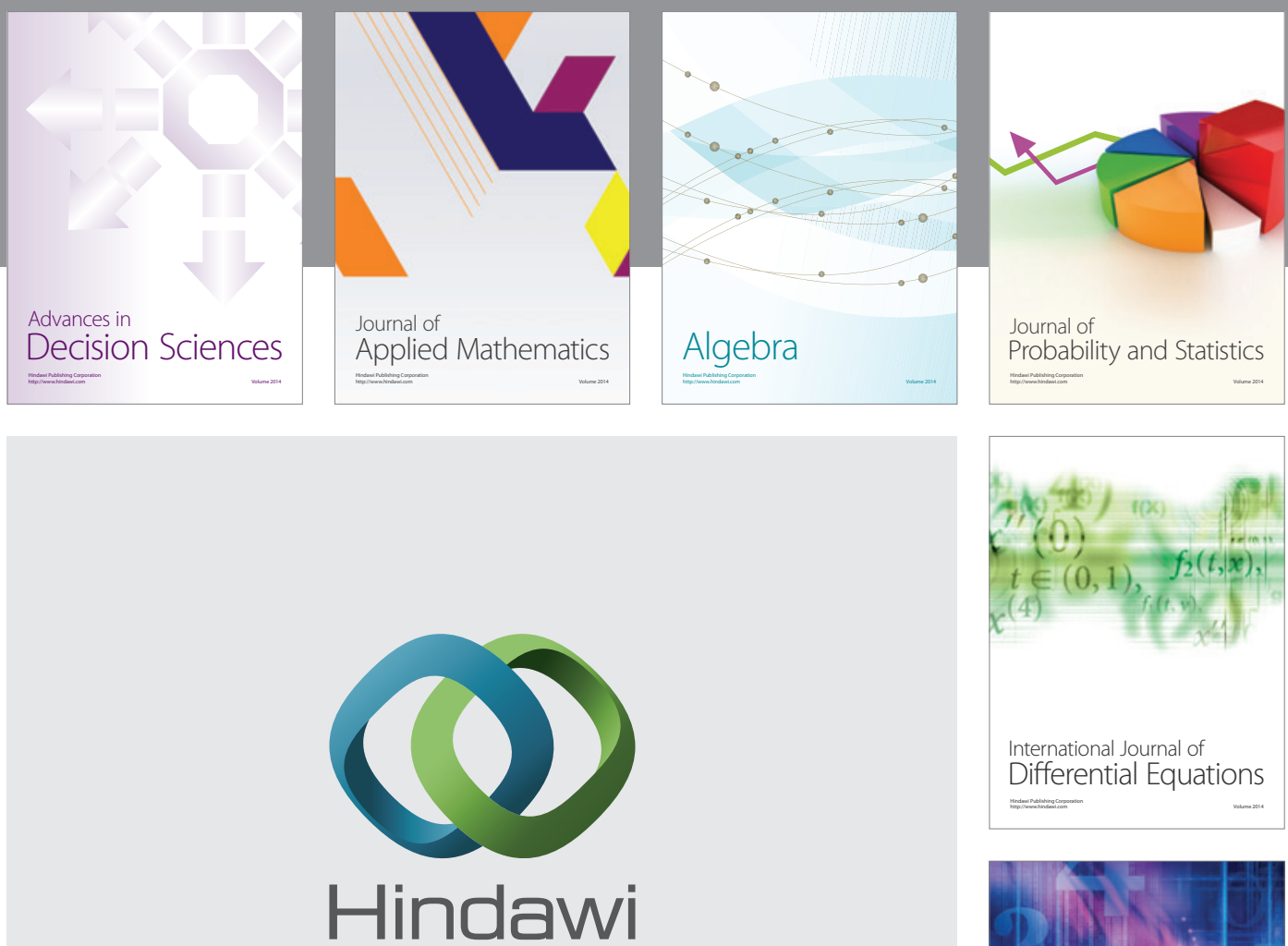

Submit your manuscripts at http://www.hindawi.com
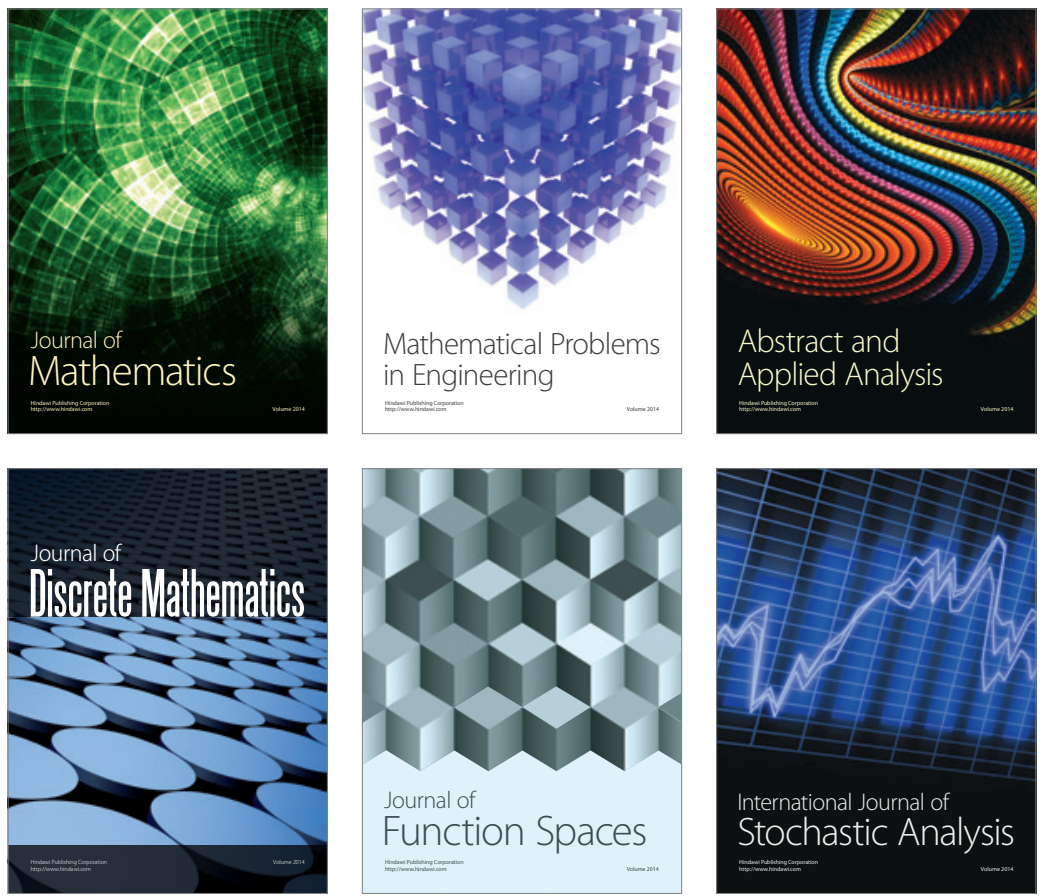

Journal of

Function Spaces

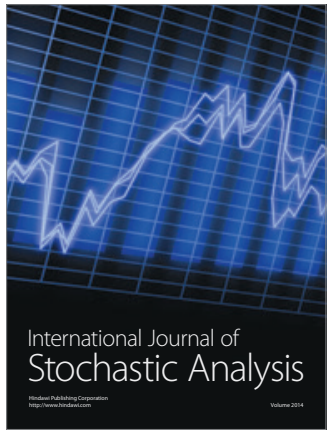

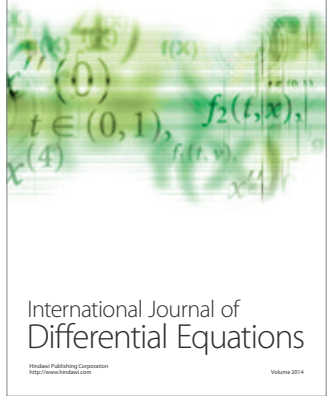
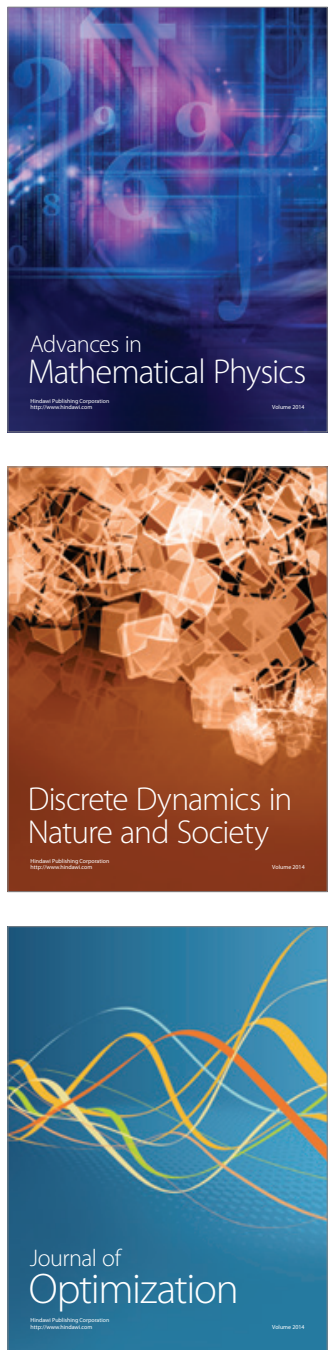